\title{
Correction: Bayesian Structured Additive Distributional Regression with an Application to Regional Income Inequality in Germany Supplement C
}

\author{
Nadja Klein, Thomas Kneib \\ Chair of Statistics \\ Georg-August-University Göttingen
}

\author{
Stefan Lang \\ Department of Statistics \\ University of Innsbruck
}

\author{
Alexander Sohn \\ Chair of Statistics \\ Georg-August-University Göttingen
}

In the following, we present the corrected results from the log-normal analysis of conditional incomes. As pointed out in the correction note (Klein et al., 2015a), the main outcome is that the log-normal distribution is closer to (but still slightly worse than) the gamma distribution but now outperforms the inverse Gaussian distribution in terms of fit and predictive ability.

Figure C1 shows the quantile residuals for the full log-normal model LN_M1 (topright panel in Figure 2 of the original paper). Visually, the results are basically indistinguishable such that the interpretation from the main paper still holds.

Table C1 reports the corrected values for the deviance information criterion and the proper scoring rules for the log-normal models LN_M1, LN_M2, LN_M3 (first three rows of Table 2 of the original paper). Here we find that the results for the deviance information criterion remain mostly unchanged with only small changes while the scores are now between the gamma and the inverse Gaussian model. As a consequence, smallest scores are obtained with the inverse Gaussian model. In summary, the Dagum model would still be preferred while log-normal and gamma are close to each other and the inverse Gaussian model clearly performs worst.

\begin{tabular}{l|ccccc}
\hline \hline Distribution & DIC & Quadratic score & Logarithmic score & Spherical score & CRPS \\
\hline LN_M1 & 179,599 & 0.155 & -2.200 & 0.388 & -1.286 \\
LN_M2 & 181,163 & 0.149 & -2.211 & 0.383 & -1.295
\end{tabular}

Table C1: Correction of the first three rows of Table 2; DIC values (calculated based on the complete data set) and average scores obtained from ten-fold cross validation.

Figure C2 shows the quantile decomposition of the continuous ranked probability score (Figure 3 of the original paper) for the full models. Note that the curves shown for gamma, inverse Gaussian and Dagum distribution actually remain completely unchanged compared to the original paper. Still, the curve for the log-normal changes dramatically such that now all lines are pretty close to each other. Again, the lognormal results lie between those of the gamma distribution and the inverse Gaussian distribution, such that the Dagum distribution ranks first while the inverse Gaussian distribution performs worst.

The corrected quantile residuals for the full models LN_M2, LN_M3 are given in Figure C3 (second row of Figure A1 in Supplement A (Klein et al., 2015b) to the 


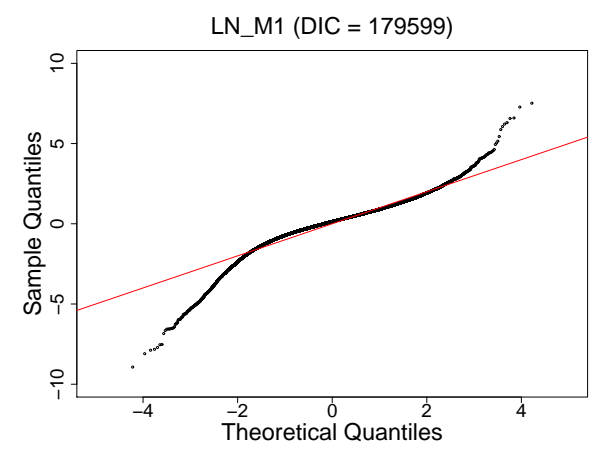

Figure C1: Correction of the topright panel of Figure 2: Quantile residuals for the full model LN_M1.

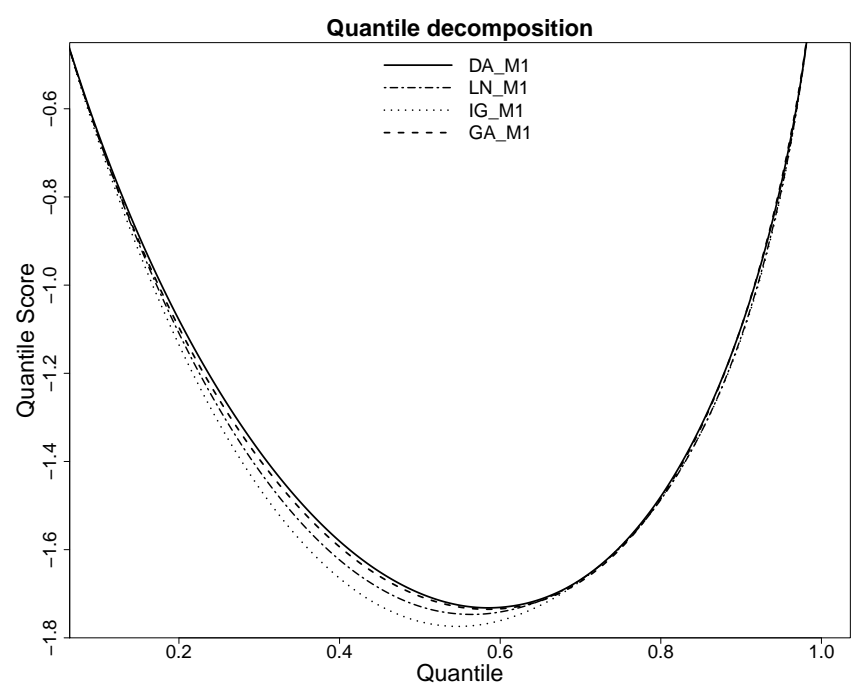

Figure C2: Correction of Figure 3: Quantile decomposition of the CRPS in the full models DA_M1, LN_M1, IG_M1, GA_M1.

original paper). Figure C4 shows the probability integral transforms for the full model LN_M1 (topright panel of Figure A2 in Supplement A (Klein et al., 2015b) to the original paper). Again, the quantile residuals (and also the probability integral transforms) are visually indistinguishable from the ones in the original paper.

Finally, Figure C5 depicts individual score contributions for the full model LN_M1 (topright panel of Figure A3 in the original paper). Note that the individual score contributions for DA_M1, IG_M1, GA_M1 are identical to the ones in the original Figure A3. As before, the differences between log-normal and gamma distribution are much smaller and now the inverse Gaussian distribution performs worst. 

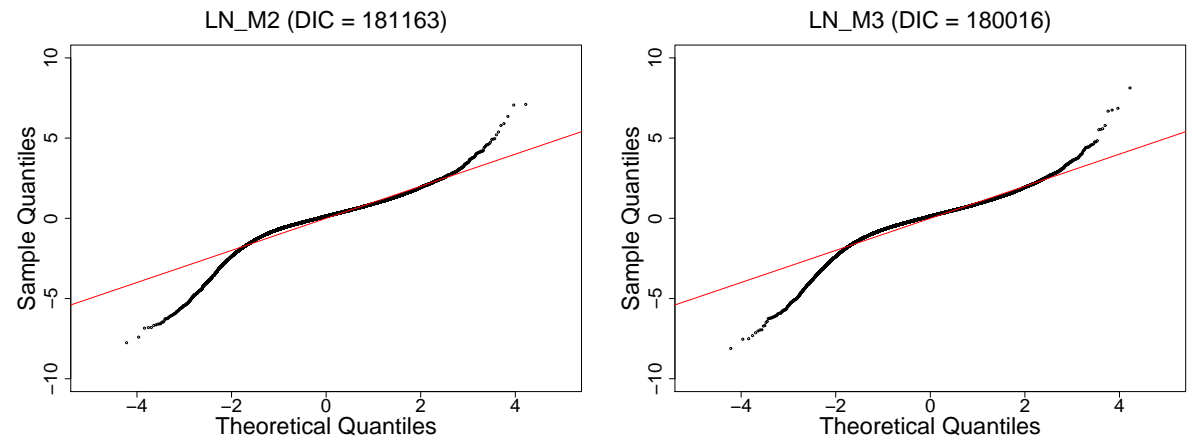

Figure C3: Correction of the second row of Figure A1: Quantile residuals for the models LN_M2, LN_M3. Model M2 corresponds to the one in which the parameter $\sigma^{2}$ is estimated as constant. In model M3, the random effects for year and region have been removed compared to M1.

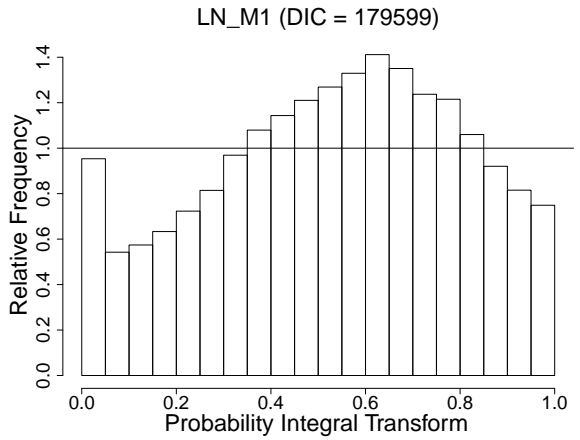

Figure C4: Correction of Figure A2: Probability integral transforms for the full model LN_M1 (topright). 

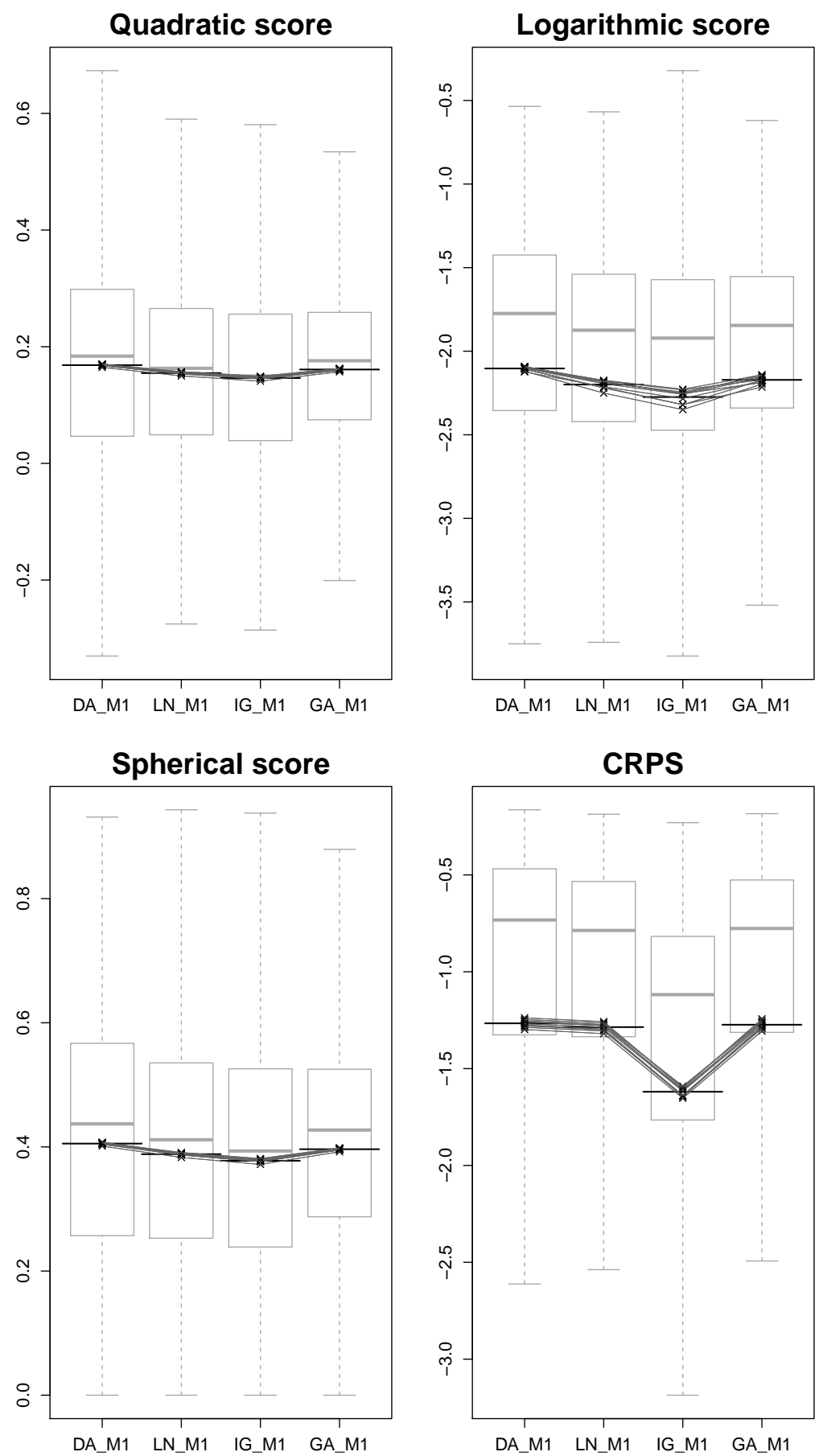

Figure C5: Correction of Figure A3: Boxplots (outliers exceeding 1.5 times the interquartile range are omitted) of individual score contributions for the whole data set. Predictions have been obtained by ten fold cross validation. The black crosses indicate average scores in each fold and are connected by grey lines between the four models. The models under consideration in each graph are DA_M1, LN_M1, IG_M1, GA_M1 (on the X-axes). On the y-axes values of the quadratic score (topleft), logarithmic score (topright), spherical score (bottomleft) and the CRPS (bottomright) are shown. Grey lines in the boxes are the median scores, the black lines the average scores documented in Table 2. 


\section{References}

Klein, N., Kneib, T., Lang, S. and Sohn, A. (2015a). Correction: Bayesian structured additive distributional regression with an application to regional income inequality in Germany.

Klein, N., Kneib, T., Lang, S. and Sohn, A. (2015b). Supplement to 'Bayesian structured additive distributional regression with an application to regional income inequality in Germany'. DOI:10.1214/15-AOAS823SUPPA. 